\title{
Gene VIII-Based, Phage-Display Vectors for Selection Against Complex Mixtures of Ligands
}

BioTechniques 24:294-301 (February 1998)

\section{Karin Jacobsson and Lars Frykberg}

Swedish University of Agricultural Sciences, Uppsala, Sweden

\begin{abstract}
Selection of shotgun phage-display libraries against complex mixtures of components, such as cells or sera, may result in a high number of nonspecifically binding phage. Consequently, correct interactions may be difficult to identify. To enable discrimination between faithful and nonspecific interactions, a set of eight different gene VIII-based, phage-display vectors were constructed. All vectors contain a "universal" screening tag positioned in such a way that it is only expressed when the inserted DNA encodes an open reading frame, which corrects a shift of reading frames in the vector. A Staphylococcus aureus shotgun phagedisplay library was made in a stoichiometric mixture of all vectors. After affinity-selection against $\operatorname{Ig} G$, one vector completely outcompeted the others. This vector contains the promoter and signal sequence from the gene encoding staphylococcal protein A and one suppressible stop codon immediately upstream of gene VIII. An increase in the frequency of clones expressing the affinity tag in all pannings correlated with selection for ligand-binding clones. This enables detection of putatively correct clones after selection of a shotgun phagedisplay library both against purified ligands and more complex materials like calf serum.
\end{abstract}

\section{INTRODUCTION}

Since its introduction in 1985 (17), phage display has developed into a very useful technique for studying proteinprotein interactions. The amino acids involved in an interaction between two molecules can be identified by using random peptide libraries (RPL) $(1-3,5,16)$. It is also possible to isolate short peptides that mimic interactions occurring in nature, such as peptides that bind to concanavalin (Con) A or streptavidin $(3,12,15)$.

We have previously shown that shotgun phage display can be used to clone ligand binding domains in prokaryotic receptors by affinity-selection against the ligand $(7,8)$. As a model bacterium, we chose Staphylococcus aureus, a pathogen responsible for a wide variety of diseases in humans and animals, such as endocarditis, osteomyelitis, wound sepsis and mastitis (4). This bacterium produces several extracellular virulence factors such as alpha-, beta-, gamma- and delta-toxins, toxic shock syndrome toxin (TSST), enterotoxins, leucocidin, proteases, coagulase and clumping factor (6). S. aureus also expresses several different surface receptors (adhesins), which interact with host matrix proteins such as fibronectin, vitronectin, collagen, laminin, elastin and bone sialoprotein (13). In addition, staphylococci can bind several serum proteins, e.g., $\mathrm{IgG}$, fibronectin, fibrinogen and trombospondin (13). Some of the genes encoding these bacterial proteins are not yet cloned, and most likely, more interactions between the host and bacteria are to be identified. The likely importance of such proteins in host/bacterial interactions prompted us to improve methods for their identification.

A shotgun phage display library, made by insertion of randomly fragmented DNA from $S$. aureus into a gene III-based phagemid vector was affinity-selected against IgG and fibronectin (Fn), resulting in 5\%-40\% positive clones (7). In a gene VIIIbased phagemid system (multi-valent display of the fusion protein), this number increased to $75 \%-100 \%$ (8). However, panning against more complex ligands, like cells or immune sera resulted in a background of nonspecifically binding phage (K. Jacobsson and L. Frykberg, unpublished observations). During the development of these cloning systems, an unusual feature was observed: both the gene III and the gene VIII phage-display systems exhibit a shift in reading frame between the inserted DNA and the coding regions within the vector. In the gene III system, the shift occurs at the transition between the 3' end of the insert and the vector; whereas, in the gene VIII system, the shift-site is located at the transition between the signal-sequence in the vector and the $5^{\prime}$ end of the insert $(7,8)$. Accordingly, in both cases, ribosomal slippage is required for expression of the fusion protein. Most likely, this apparent selection for inserts in the "wrong" reading frame is a natural way for the system to optimize the production of viable phage by down-regulating the expression of the coat-fusion protein. Unfortunately, in the gene-VIII system, this results in an expression 
level of the encoded insert too low for detection in a screening assay with a peroxidase-labeled ligand. This can create problems, since yielding low frequences of positive clones may require a screening system to identify binding clones. In addition, panning against more complex materials consisting of a mixture of different ligands, like cells and serum, will result in an enrichment of a high number of clones reacting with different, unknown ligands. Thus, no ligand-specific screening system can be used. Therefore, we have developed a set of vectors with a "universal" screening tag out-of-frame with the signal sequence, but in-frame with gene VIII. This design ensures that clones with inserts containing an open reading frame (ORF) that corrects the frameshift can be detected with labeled human serum albumin (HSA). This means that an increasing fraction of clones expressing the tag will correlate to a selection for the displayed polypeptides. Such an approach can only be used when DNA fragments of random, variable length are inserted into a phage-display vector. In addition, a number of precautions have been taken to avoid selection for clones with inserts out of reading frame, which would hamper detection of the tag.
First, sequences suspected to promote ribosomal slippage in pHEN1 and pG8H6 $(7,8)$ were omitted. Second, 0-3 suppressible stop codons were introduced between the tag and gene VIII to obtain varying expression levels of the full-length fusion protein, but ensuring a high expression of insert and tag (Figure 1). Third, a second set of vectors identical to the ones described above, except for a substituted promoter and signal sequence [from the gene encoding staphylococcal protein A (spa)], was constructed (pG8SPA0-3; Figure 1).

A shotgun phage-display library was made in a stoichiometric mixture of all eight vectors, and panning against IgG was used to determine which vector was able to outcompete the others. This library was also affinity-selected against newborn calf serum (NCS). Our data showed that positive clones with affinity for serum components could be identified using labeled HSA, i.e., expression of the screening tag.

\section{MATERIALS AND METHODS}

\section{Bacterial Strains and Helper Phage}

E. coli MC1061 was used for con- struction of the vectors, and E. coli strain TG1 was used for construction of libraries and production of phage stocks. Phage R408 (Promega, Madison, WI, USA) was used as helper phage. E. coli transformants were grown in LB medium supplied with $1 \%$ glucose and $50 \mu \mathrm{g} / \mathrm{mL}$ ampicillin.

\section{Construction of the Vectors}

All DNA manipulations were performed using standard methods (14), except for ligations for which ReadyTo-Go ${ }^{\mathrm{TM}}$ T4 DNA Ligase (Pharmacia Biotech, Uppsala, Sweden) was used. Modification and restriction enzymes were from Amersham International (Little Chalfont, Bucks, England, UK) or MBI Fermentas AB (Vilnius, Lithuania). All oligonucleotides were synthesized by Pharmacia Biotech.

The pG8SPA- and pG8LP-phagemid vectors were constructed from pG8H6 (8). Primers Gen83 and G8Bam were used to amplify gene VIII from pG8H6 and simultaneously introducing a BamHI site in front of the gene. The fragment encoding the HSAbinding domain was amplified from pSZG40 (10) using the primer NcoAlb, containing the SmaI and SnaBI sites for blunt-end cloning, and either AlbStO,

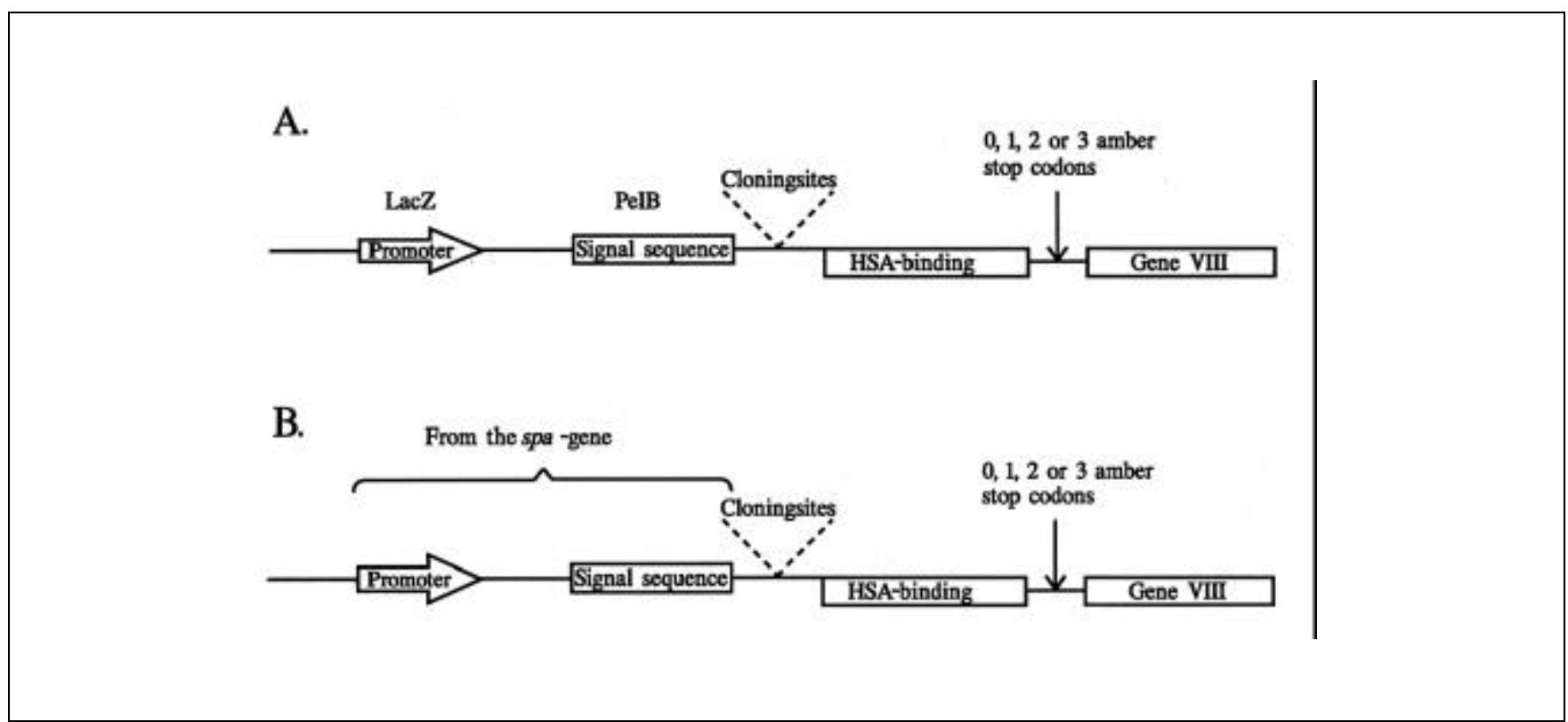

Figure 1. Schematic drawing of (A) the pG8PL and (B) the pG8SPA vectors. The signal sequence and the HSA-binding domain are not in the same reading frame, and thus no HSA-binding polypeptide is produced unless an insert has corrected the shift in reading frame. Cloning sites present in all vectors: NcoI, SnaBI and SmaI/XmaI. 
Table 1. Deoxyoligoribonucleotides Used in the Construction of the pG8LP- and PG8SPA-Series of Phagemid Vectors and/or Used in Hybridizations and Nucleotide Sequencing of the Inserts

\begin{tabular}{|lll|}
\hline NcoAlb & Constr/hyb & $5^{\prime}$-gat ccc atg gca gta cgt acc cgg ggc gga cat tac agg agc agc ctt gt-3' \\
AlbSt0 & Constr/hyb & $5^{\prime}$-gat cgg atc cga cct cag aac tcg gta gag c-3' \\
AlbSt1 & Constr/hyb & $5^{\prime}$-gat cgg atc cct aga cct cag aac tcg gta gag ctg a-3' \\
AlbSt2 & Constr & $5^{\prime}$-gat cgg atc cct act aga cct cag aac tcg gta gag ctg ata-3' \\
AlbSt3 & Constr & $5^{\prime}$-gat cgg atc cct act act aga cct cag aac tcg gta gag ctg ata aaa-3' \\
Pranc & Constr & $5^{\prime}$-cga tcc cat ggt cat cgt gtt gcg cag cat tt-3' \\
SapPr & Constr & $5^{\prime}$-gat cag cgg aag agc cgg ttt taa gcc ttt tac t-3' \\
G8Bam & Constr & $5^{\prime}$-ata ggg atc cga ggg tga cga tcc cgc aaa-3' \\
Gen83 & Constr & $5^{\prime}$-ttt ccc gaa ttc atc ggt tta tca gct tgc ttt cg-3' \\
Salead & $5^{\prime}$-tat ctg gtg gcg taa cac ctg ct-3' \\
Pel & Hyb/sequen & $5^{\prime}$-cct att gcc tac ggc agc cgc tgg-3' \\
H2Stop & Hyb/sequen & $5^{\prime}$-tga ggt cta gta ggg at-3' \\
H3Stop & Hyb & $5^{\prime}$-agg tct tag tag tag gga-3' \\
Albp & Hyb & $5^{\prime}$-gcc ata ctg ctt tag ttc att gat $-3^{\prime}$ \\
Sites for the restriction enzymes used in the cloning procedure are underlined.
\end{tabular}

Table 2. Number of Eluted Phagemid Particles (cfu) and Fraction of IgG- and HSA-Binding Clones after Panning Against Human IgG.

\begin{tabular}{|lccc|}
\hline & Eluate $(\mathbf{c f u})^{\mathbf{a}}$ & HSA Bind & IgG Bind $\mathbf{b}$ \\
\hline 1st panning & $1 \times 10^{5}\left(1 \times 10^{5}\right)$ & $20(39)$ & $29(44)$ \\
2nd panning & $3 \times 10^{7}\left(5 \times 10^{7}\right)$ & $58(67)$ & $74(82)$ \\
3rd panning & nd $\left(2 \times 10^{8}\right)$ & nd (62) & nd (92) \\
aNumber of colony-forming units. Figures in parenthesis represent the second \\
experiment. \\
bPercentage ligand-binding clones of 150 screened. \\
nd = not done.
\end{tabular}

AlbSt 1, AlbSt 2 or AlbSt 3 to introduce $0,1,2$ or 3 amber stop codons, respectively. A fragment containing the promoter region and the signal sequence from the spa gene was amplified from the plasmid pSPA15 (18) using primers Pranc and Sap1pr. The fragments obtained were cut with BamHI/EcoRI, NcoI/BamHI and SapI/NcoI, respectively. First, the pG8LP-series was constructed by digesting pG8H6 with EcoRI/NcoI, followed by treatment with calf intestine alkaline phosphatase (CIAP). Subsequently, the fragments encoding gene VIII and the HSA-binding domain were ligated into the vector, and the ligation mixture transformed into E. coli MC1061. Plasmids prepared from ampicillin-resistant colonies were analyzed by restriction digestion. Plasmids yielding the expected restriction pattern were further characterized by nucleic acid sequence determination. Next, the vector pG8LP0-3 was digested with SapI and NcoI followed by CIAP-treatment. The spa fragment was ligated into the respective vector, and the ligation mixtures were transformed into E. coli. Plasmids were prepared from ampicillin-resistant colonies and analyzed as described above. A polymerase chain reaction (PCR)-induced point mutation in the protein A signal sequence, resulting in an amino acid exchange from Lys to Arg in the third position, was found in all four pG8SPA vectors.

\section{Construction of the Library}

The library was constructed as described previously $(7,8)$. In short, a mixture of $10 \mu \mathrm{g}$ of each of the eight vectors was digested with $S n a \mathrm{BI}$ and dephosphorylated. Eight micrograms of the vector mixture were ligated with $8 \mu \mathrm{g}$ of $S$. aureus fragments (300-2000 bp) in 13 tubes of Ready-To-Go Ligase (Pharmacia Biotech). The ligated material was phenol- and chloroform-extracted, ethanol-precipitated and dissolved in $20 \mu \mathrm{L}$ of $\mathrm{H}_{2} \mathrm{O}$. Twenty $1-\mu \mathrm{L}$ aliqouts of the ligation mixture were electrotransformed into E. coli TG1, and the transformed cells were grown overnight in $200 \mathrm{~mL} \mathrm{LB}$ medium supplied with $1 \%$ glucose and $50 \mu \mathrm{g} / \mathrm{mL}$ ampicillin. A 4-mL aliqout of the overnight culture was infected with helper phage (multiplicity of infection [MOI] 20), mixed with $100 \mathrm{~mL} 0.5 \%$ soft agar and poured onto $20 \mathrm{LA}$ plates (LB medium with $1.5 \%$ agar and 50 $\mu \mathrm{g} / \mathrm{mL}$ ampicillin). After overnight incubation, the phages were eluted from the soft agar.

\section{Panning Procedures}

Pannings were carried out as described $(7,8)$ with some minor modifications. Microwell ${ }^{\circledR}$ plates (MaxiSorp ${ }^{\mathrm{TM}}$; Nalge Nunc International, Copenhagen, Denmark) were coated with human IgG (Kabivitrum, Stockholm, Sweden) at a final concentration of $100 \mu \mathrm{g} / \mathrm{mL}$ in $0.05 \mathrm{M}$ sodium car- 
bonate, $\mathrm{pH}$ 9.7, and blocked with PBS$\mathrm{T}$ (phosphate-buffered saline with $0.05 \%$ Tween ${ }^{\circledR} 20$ ). NCS (Life Technologies, Inchinnan, Scotland, UK) was diluted 20 times in coating buffer. The library and the stocks used for repannings were pre-incubated with 0.1 $\mathrm{mg} / \mathrm{mL}$ HSA (Sigma Chemical, St. Louis, MO, USA) for 15 min before addition to the wells to inhibit nonspecific binding to potentially contaminating HSA. In some pannings against NCS, the library/stock was pre-incubated with human $\operatorname{IgG}(100 \mu \mathrm{g} / \mathrm{mL})$ to avoid enrichment for protein A. After panning for $3 \mathrm{~h}$ at room temperature, the wells were rinsed 30 times with PBS-T, bound phage were eluted with $200 \mu \mathrm{L} 50 \mathrm{mM}$ Na-citrate $/ 140 \mathrm{mM}$ $\mathrm{NaCl}, \mathrm{pH} 2.0$, followed by neutralization with $25 \mu \mathrm{L} 2 \mathrm{M}$ Tris-HCl, $\mathrm{pH}$ 8.7. The eluate was used to infect $E$. coli TG1 and plated for determining the number of phagemids and for amplification as described previously (8). To determine how the phagemids replicate in the absence of affinity-selection, a $10-\mu \mathrm{L}$ portion of the library was re-amplified twice.

After each panning against IgG, 150 colonies were picked to 9 plates (LB medium with $1.5 \%$ agar and $50 \mu \mathrm{g} / \mathrm{mL}$ ampicillin) in identical patterns. All eight vectors without inserts were included as controls. After pannings against calf serum, the colonies were picked to 3 or 4 plates in identical patterns.

\section{Screening}

Colonies from all pannings were screened for binding of horseradish peroxidase (HRP)-labeled HSA ( $5 \mu \mathrm{g} / \mathrm{mL})$ or IgG $(5 \mu \mathrm{g} / \mathrm{mL})$ as described (7). After pannings against NCS, some filters were screened for fibronectin-binding clones using human fibronectin (10 $\mu \mathrm{g} / \mathrm{mL}$ ) and rabbit anti-human fibronectin antibodies, followed by detection with HRP-labeled goat anti-rabbit antibodies (all from Sigma Chemical) according to the manufacturer's instructions.

\section{Hybridizations}

Colonies were transferred to nitrocellulose filters and processed accord- ing to standard procedures (14). Hybridizations were carried out at temperatures $5^{\circ}-10^{\circ} \mathrm{C}$ below melting temperature $\left(\mathrm{T}_{\mathrm{m}}\right)$, and washings were done in $0.2 \%$ sodium dodecyl sulfate (SDS) $/ 2 \times$ standard saline citrate (SSC) or $0.5 \%$ SDS $/ 6 \times$ SSC $(20 \times \mathrm{SSC}=3.0 \mathrm{M} \mathrm{NaCl}$ and $0.3 \mathrm{M} \mathrm{Na}$-citrate) at $5^{\circ} \mathrm{C}$ below $\mathrm{T}_{\mathrm{m}}$. The specificity was confirmed by exposure of wet filters to a PhosphorImager ${ }^{\circledR}$ (Molecular Dynamics, Sunnyvale, CA, USA) and, if background signals remained, the washing was continued at temperatures increased in steps of $1^{\circ} \mathrm{C}$.

\section{Nucleotide Sequencing of the Displayed Inserts}

Plasmid DNA was prepared using Wizard $^{\mathrm{TM}}$ Minipreps (Promega), and the sequence of the inserts was determined as described previously (8), using primers Albp and Salead or Pel (Table 1). The PC-gene program (IntellliGenetics, Mountain View, CA, USA) was used for handling of the sequences obtained.

\section{RESULTS AND DISCUSSION}

\section{Construction of the Vectors}

We reported earlier that shotgun phage-display cloning of bacterial adhesins results in $75 \%-100 \%$ correct clones after two pannings in a gene VIII-based system (8). However, this result was obtained after panning against a pure ligand, and the binding by the receptor was mediated by a short domain. Panning against more complex materials, like cells or immune serum, resulted in a high background of nonspecifically binding phage (K. Jacobsson and L. Frykberg, unpublished results). In addition, the shifts in reading frame found in almost all clones after affinity-selection hamper the identification of positive clones with a labeled ligand. Therefore, we decided to construct new improved gene VIII-based phage display vectors, which should be able to express the inserted DNA in correct reading frame. Hence, the sequences encoding the c-myc tag and the histidine tail, which we believe promote ribosomal slippage in pHEN1 and 
pG8H6, respectively, were omitted. In addition, an increasing number of suppressible amber stop codons $(0,1,2$ or 3 ) were placed in front of gene VIII, which is expected to give a reduced expression of the fusion protein. This reduced expression should circumvent the selection for clones with inserts that are out-of-reading-frame. Furthermore, a screening tag was introduced in such a way that it enables detection of putative positive clones irrespective of the ligand used in the panning. The HSAbinding tag was introduced out-ofreading-frame with the signal sequence, but in-frame with gene VIII, and with a cloning site positioned immediately upstream of the tag (vectors pG8PL0-3; Figure 1A). Thus, an appropiately inserted DNA fragment with an ORF will restore the ORF and, accordingly, the tag will be expressed. The amber stop codons were placed downstream of the screening tag so that the expression of the insert and tag seg-

Table 3. Fraction of Clones Assigned to the Different Vectors after Pannings Against IgG or After Repeated Amplifications of the Library in the Absence of Any Selection

\begin{tabular}{|c|c|c|c|c|c|c|c|c|}
\hline & \multicolumn{4}{|c|}{ pG8SPA Vectors } & \multicolumn{4}{|c|}{ pG8LP Vectors } \\
\hline & $0^{a}$ & $1^{a}$ & $2^{a}$ & $3^{a}$ & $0^{a}$ & $1 \mathrm{a}$ & $2^{a}$ & $3^{a}$ \\
\hline $\lg G 1 s t$ & $19(15)$ & $29(44)$ & $24(28)$ & $6(7)$ & $4(0)$ & $7(5)$ & $5(0)$ & $6(2)$ \\
\hline 2nd & $19(18)$ & $56(65)$ & $16(17)$ & $2(0)$ & $1(0)$ & $3(1)$ & $1(0)$ & $1(0)$ \\
\hline $3 r d$ & $17(17)$ & $74(78)$ & $5(5)$ & $1(1)$ & $0(0)$ & $1(0)$ & $0(0)$ & $2(0)$ \\
\hline Uns.1st & \multicolumn{4}{|c|}{76} & \multicolumn{4}{|c|}{24} \\
\hline 2nd & 22 & 22 & 23 & 20 & 4 & 3 & 1 & 5 \\
\hline $3 r d$ & 28 & 26 & 17 & 25 & 1 & 1 & 1 & 1 \\
\hline
\end{tabular}

aNumber of stop codons in the vector.

Figures show the percentage of clones contained in the respective vector of all 150 colonies screened. Figures in parenthesis show the percentage of clones contained in the respective vector of the IgG-binding clones among the 150 colonies screened.

ment should remain high. Thereby, it should be possible to detect expression of the insert and/or the tag. Earlier pannings of $S$. aureus shotgun phage-dis- play libraries against IgG resulted in a significant fraction of clones expressed from the spa promoter, in which the protein A signal sequence promoted 
Table 4. Number of Eluted Phagemid Particles after Panning Against NCS and the Frequency of Bacterial Colonies Expressing Affinity for IgG, HSA or Fibronectin

\begin{tabular}{|c|c|c|c|c|c|}
\hline Panning & Wash (cfu)a & Eluate $(\mathrm{cfu})^{\mathrm{a}}$ & HSA Bindb & IgG Bind $b$ & Fn Bind $b$ \\
\hline $1 \mathrm{st}$ & $6 \times 10^{4}\left(1 \times 10^{4}\right)$ & $4 \times 10^{4}\left(3 \times 10^{4}\right)$ & $3(5)$ & $0.7(1)$ & nd (7) \\
\hline 2nd & $1 \times 10^{5}\left(3 \times 10^{4}\right)$ & $3 \times 10^{5}\left(3 \times 10^{5}\right)$ & $18(47)$ & $5(23)$ & nd (17) \\
\hline $3 r d$ & $2 \times 10^{5}\left(2 \times 10^{5}\right)$ & $1 \times 10^{7}\left(7 \times 10^{7}\right)$ & $44(59)$ & $89(67)$ & nd $(0)$ \\
\hline 3rd inhe & & & $51(\mathrm{nd})$ & $0.7(\mathrm{nd})$ & $19(\mathrm{nd})$ \\
\hline \multicolumn{6}{|c|}{$\begin{array}{l}\text { aNumber of colony-forming units per } \mathrm{mL} \text {. Two separate experiments are shown, the second of which is shown in parenthesis } \\
\text { bPercentage ligand-binding clones of } 150 \text { screened. } \\
\text { cThird panning repeated in the presence of IgG. } \\
\text { nd = not done. }\end{array}$} \\
\hline
\end{tabular}

transport of the coat protein to the membrane (Reference 8 and unpublished observation [K. Jacobsson and L. Frykberg]). This result suggested that the spa promoter and signal sequence might be suitable in a phage-display vector. Therefore, a set of four vectors was constructed, where the lac promoter and pelB leader in pG8PL0-3 were exchanged for the spa promoter and signal sequence (Figure 1B).

\section{Construction of a Library in a Mixture of Eight Vectors}

All eight vectors, mixed in stoichiometric amounts, were used for construction of a $S$. aureus shotgun phagedisplay library. The final library consisted of $3 \times 10^{8}$ independent clones, $88 \%$ of which contained an insert, and had a titre of $3 \times 10^{10}$ colonyforming units $(\mathrm{cfu}) / \mathrm{mL}$. After ligation, the frequency of clones that had obtained HSA-binding fortuitously, i.e., carried an inserted DNA fragment with an ORF and with a length that corrected the shift in reading frames in the vector, was approximately $3 \%$ (data not shown). In the propagated library, after infection with helper phage, the frequency of HSA-binding clones had decreased to approximately $1 \%$. Thus, an increase to more than 1\% HSA-binding clones after panning should indicate a selection for the displayed polypeptide.

\section{After Panning Against IgG, Clones Expressing the Activated Tag Correlate with Positive Clones}

To confirm the correlation between selection for ligand-binding clones and an increasing fraction of HSA-binding clones, the library was panned against human $\mathrm{IgG}$ in two separate experiments. After each panning, 150 colonies were characterized with respect to binding of HRP-labeled IgG and HSA (Table 2). After three consecutive pannings, the number of bound phagemid particles had increased 1000fold. After the third panning, 92\% of the bacterial colonies obtained from the eluted phagemids could be detected with labeled $\operatorname{IgG}$, and out of these, 2/3 also bound HSA (Table 2). This indicates that $92 \%$ of the inserts are fused in-frame with the signal sequence, but a significant fraction (1/3) still showed a shift in reading frames between the inserted DNA and the HSA-binding domain. The nucleotide sequence of five IgG-positive/HSA-negative clones was determined. As expected, they showed a shift in reading frame between the insert and the tag (data not shown). Nevertheless, the reading frame shifts were found in only $33 \%$ of the clones, as determined by the ability of the bacterial colonies to bind IgG and HSA, compared with 48 out of 48 clones obtained with the previous vector (pG8H6) (8). It is possible that panning against another ligand may result in a different frequency of inserts out-of-frame depending on how the encoded polypeptide affects the E. coli cell. Labeled HSA was not uniformly bound to different colonies, which was not unexpected since different lengths of the inserted DNA might result in different expression levels and/or could be due to decreased accessibility of the HSAbinding domain due to the remainder of the expressed polypeptide. Also, it cannot be excluded that some of the weak HSA signals could be caused by a frame shift, which is efficiently corrected by ribosomal slippage.

\section{Competition Between the Vectors}

The panning experiments described above were also used for selection of the vector with superior performance. For this purpose, the signal sequence and the number of stop codons were determined in the 150 colonies characterized after each panning (Table 3). Already after one round of selection, an enrichment for the pG8SPA vectors was seen. After additional consecutive pannings, which resulted in an increasing number of IgG-binding clones, the pG8SA1 vector (spa promoter and signal sequence with one stop codon) outcompeted the others. After three pannings, $78 \%$ of the IgG-binding clones were identified as pG8SPA1 derivatives. No IgG-binding clones were found in any of the vectors with the lac promoter and pelB leader (Table 3). This result was unexpected, since the shift in reading frame found earlier must reduce the expression level of fusion protein and, hence a repressible promoter (e.g., lac promoter) was expected to be preferred. The preference for the pG8SPA vectors could conceivably depend on the signal sequence, which might be more efficient for translocation of the fusion protein to the membrane.

To determine if there was any difference in how well the phagemid vectors replicated, they were subjected to three rounds of amplification without affinity-selection. Surprisingly, the pG8SPA vectors also now outcompeted the pG8PL vectors. After three amplifications, $96 \%$ of the clones were identified 
as pG8SPA vectors, and only small differences in the number of stop codons were found (Table 3). This result suggests that the pG8SPA vectors are either more efficient in replication or in packaging of the phagemid.

\section{Panning Against Newborn Calf Serum}

The library was also panned against NCS to test whether a selection as reported above also hold true for interactions with a complex mixture of different ligands and whether the "universal screening tag" can be used to identify "correct" clones after such pannings. Note that the tag was reported not to bind bovine serum albumin (BSA) in a dot blot assay (10). However, in this multi-valent display system, the tag does react with BSA, although with an affinity much lower than for HSA. Thus, the binding to BSA can conveniently be inhibited by incubating the library with HSA before panning (data not shown). In all pannings, phage stocks were pre-incubated with HSA at a final concentration of $100 \mu \mathrm{g} / \mathrm{mL}$ for 15 min before addition to the coated wells. After three consecutive pannings against NCS in two separate experiments, the number of binding phage had increased approximately 1000 -fold (Table 4). After each panning, 150 colonies were screened for HSA-, IgGand, in some cases, for fibronectin (Fn)-binding. The frequency of HSA binding clones increased from approximately $4 \%$ after the first panning to about $50 \%$ after the third panning (Table 4). After the first panning, the fraction of IgG-binding clones was very low, but after three pannings, it ranged from $65 \%-89 \%$. In the second experiment, the number of Fn-binding clones was also determined. After the first panning, more Fn-binding than IgG-binding clones were found, but in the second and third pannings, the Fnbinding clones were outcompeted, mainly, by IgG- binding clones.

In the first experiment, almost exclusively, IgG-binding clones were recovered after three consecutive pannings. To avoid this selection, the stock used in the third panning was pre-incubated with human IgG, which reduced the fraction of IgG-binding clones from $89 \%$ to less than $1 \%$, while the fraction of HSA-binding clones was unaffected. This indicates that pre-incubation of the phage stock with free ligand efficiently blocks binding of phage to the same serum component. Thereby, an enrichment for other clones is obtained. These results clearly demonstrate that the enrichment of serum protein-binding clones can be followed as an increase in HSA-binding clones, as shown in Table 4 . In the first experiment (second panning), 15 HSA-binding clones that did not bind $\mathrm{IgG}$ were characterized by DNA sequencing. Fourteen of these contained DNA encoding Fn-binding proteins $(9,11)$. The 15 th clone contained an insert derived from sbi gene, the second IgG-binding protein in $S$. aureus $(7,19)$. Interestingly, the fragment isolated here lacks the IgG-binding domain (data not shown). The same $s b i$ fragment was found in 15 clones, judged as negative for Fn binding but positive for HSA binding after the third panning with IgG inhibition. In the second experiment, 16 clones, likewise negative for IgG and Fn binding, were sequenced. Fourteen clones contained overlapping inserts derived from the $s b i$ gene, only one of which contained the IgG-binding domain (data not shown). This strongly suggests that the SBI protein binds a second serum component beside IgG.

\section{CONCLUSIONS}

After three consecutive pannings against IgG, with the library made in a mixture of all eight vectors, the pG8SPA1 vector, i.e., spa promoter and signal sequence combined with one stop codon, had outcompeted the others. Since this result was obtained after panning against one ligand only, it cannot be excluded that other ligands would enrich for a different vector, depending on whether a lower or higher number of fusion proteins per phage particle is beneficial. Still, we suggest that the pG8SPA1 vector should be used for future construction of shotgun phage-display libraries.

Furthermore, correct clones could be identified by detection of the screening tag after affinity-selection against a complex mixture of molecules. The use of a universal affinity tag expressed only in clones containing an ORF that corrects the frame shift introduced in the vector, is extremely useful for identification of putatively correct clones. It significantly reduces the number of clones to investigate even if the background of nonspecifically binding phage to the immobilized ligands is high. Maybe more importantly, we show here that this can be done already after the first panning. This is desired, since consecutive pannings may sometimes result in enrichment for a single type of clone, as illustrated by the sequencing of 15 clones containing the same part of the sbi gene after three pannings against NCS. Similarly, three pannings against NCS, without inhibition with free $\mathrm{IgG}$, resulted in enrichment for almost exclusively IgG-binding clones. Hence, less avid interactions may remain undetected if successive enrichment steps are used.

Infection of the host by pathogenic 
bacteria involves multiple interactions. Both extracellular and surface-located bacterial proteins interact with soluble as well as cell and matrix-associated proteins of the host. The identification of such bacterial proteins are important for the understanding of the infection process. Panning against serum resulted not only in enrichment for IgG- and Fn-binding polypeptides but also identified an unexpected interaction between the SBI protein and a so far unidentified serum component.

Libraries constructed from bacterial DNA in the vectors described here, can be used for selection against the host structures with which bacteria interact, such as serum components, cells, tissues or implants removed from patients, and binding clones can be identified using the tag. The vectors and the techniques described in this paper can be directly applied to all prokaryotes and, with some modifications, also to eukaryotes.

\section{ACKNOWLEDGMENTS}

The authors are grateful to professors Martin Lindberg and Gerhart Wagner for helpful comments on the manuscript. The work was supported by the Swedish Research Council for Engineering Sciences (Grant Nos. 94-380 and 96-759) and the Immunotechnology Program founded by the Swedish National Board for Industrial and Technical Development (Grant No. P2355-19).

\section{REFERENCES}

1.Cortese, R., P. Monaci, N. Nicosia, A. Luzzago, F. Felici, G. Galfré, A. Pessi, A. Tramontano and M. Sollazzo. 1995. Identification of biologically active peptides using random libraries displayed on phage. Curr. Opin. Biotechnol. 6:73-80.

2.Cwirla, S.E., E.A. Peters, R.W. Barrett and W.J. Dower. 1990. Peptides on phage: a vast library of peptides for identifying ligands. Proc. Natl. Acad. Sci. USA 87:6378-6382.

3.Devlin, J.J., L.C. Panganiban and P.E. Devlin. 1990. Random peptide libraries: a source of specific binding molecules. Science 249:404-406.

4.Easmon, C.S.F. and C. Adlam. 1983. Staphylococci and staphylococcal infections, Vols. 1 and 2. Academic Press, London.

5.Hoess, R. 1993. Phage display of peptides and protein domains. Curr. Opin. Struct. Biol. 3:572-579.
6.Iandolo, J.J. 1989. Genetic analysis of extracellular toxins of Staphylococcus aureus. Annu. Rev. Microbiol. 43:375-402.

7.Jacobsson, K. and L. Frykberg. 1995. Cloning of ligand-binding domains of bacterial receptors by phage display. BioTechniques 18:878-885.

8.Jacobsson, K. and L. Frykberg. 1996. Phage display shot-gun cloning of ligand-binding domains of prokaryotic receptors approaches $100 \%$ correct clones. BioTechniques 20:10701081.

9.Jönsson, K. Fibronectin-binding proteins of Staphylococcus aureus. Swedish University of Agricultural Sciences, Uppsala Sweden. Dissertation Series Rapport-52; 1992. Thesis.

10.Jonsson, H., H. Lindmark and B. Guss. 1995. A protein G-related cell surface protein in Streptococcus zooepidemicus. Immunology 63:2968-2975.

11.Jönsson, K., C. Signäs, H.-P. Müller and M. Lindberg. 1991. Two diffferent genes encode fibronectin binding proteins in Staphylococcus aureus. The complete nucleotide sequence and characterization of the second gene. Eur. J. Biochem. 292:1041-1048.

12.Oldenburg, K.R., D. Loganathan, I.J. Goldstein, P.G. Schultz and M.A. Gallop. 1992. Peptide ligands for a sugarbinding protein isolated from a random peptide library. Proc. Natl. Acad. Sci. USA 89:5393-5397.

13.Patti, J.M., B.L. Allen, M.J. McGavin and M. Höök. 1994. MSCRAMM-mediated adherence of microorganisms to host tissue. Annu. Rev. Microbiol. 48:585-617.

14.Sambrook, J., E.F. Fritsch and T. Maniatis. 1989. Molecular Cloning: A Laboratory Manual, 2nd ed. CSH Laboratory Press, Cold Spring Harbor, NY.

15.Scott, J.K., D. Loganthan, R.B. Easley, X. Gong and I.J. Goldstein. 1992. A family of cocanavalin A-binding peptides from a hexapeptide epitope library. Proc. Natl. Acad. Sci. USA 89:5398-5402.

16.Scott, J.K. and G.P. Smith. 1990. Searching for peptide ligands with an epitope library. 249:386-390.

17.Smith, G.P. 1985. Filamentous fusion phage: novel expression vectors that display clones antigens on the virion surface. Science 228:1315-1317.

18.Uhlén, M., B. Guss, B. Nilsson, F. Götz and M. Lindberg. 1984. Expression of the gene encoding protein A in Staphylococcus aureus and coagulase-negative staphyloccoci. J. Bacteriol. 159:713-719.

19.Zhang, L., K. Jacobsson, J. Vasi, M. Lindberg and L. Frykberg. A second IgG-binding protein in Staphylococcus aureus. Microbiology (In press).

Received 26 June 1997; accepted 19 August 1997.

Address correspondence to:

Lars Frykberg

Box 7025

S-750 07 Uppsala, Sweden

Internet: lars.frykberg@mikrob.slu.se 